\title{
Decoupling and Matching Strategies for Compact Antenna Arrays
}

\author{
F. Estevão S. Pereira, Josef A. Nossek, Life Fellow, IEEE, and F. Rodrigo P. Cavalcanti
}

\begin{abstract}
Since the MIMO technology presents antenna arrays that can be formatted with tens to hundreds antenna elements, several effects arise due to this array structure, such as mutual coupling and impedance matching among antenna elements in the array. The treatment of these issues seeks to solve problems such as the performance degradation of communication systems. In this context, this paper proposes and evaluates three strategies of joint decoupling and impedance matching networks (DMN) for antenna arrays. The first method, called DMN with Lumped Elements (DMN-LE), performs the decoupling and impedance matching steps with capacitors and inductors. The second method, called DMN with Ring Hybrid (DMN-RH), utilizes a microstrip line in a ring format. The third method, called Networkless Decoupling and Matching (NDM), brings a concept of decoupling without the presence of a network itself, which enables modeling an antenna array that performs DMN operations in a simplified and compact manner. A comparison of the methods is performed both analytically and via computer simulations. The figure-of-merit for this comparison is the antenna bandwidth, for which both matching and decoupling are measured against $S$-parameters below a given threshold. We conclude that the third method is a promising new alternative approach.
\end{abstract}

Index Terms-antenna array, MIMO, decoupling network, impedance matching.

\section{INTRODUCTION}

A NTENNA arrays have been used in various applications and have become an important tool to achieve high spectral efficiency in wireless communications. Its use brings to the communication systems an increase in performance in terms of capacity and reliability.

The Multiple-Input Multiple-Output (MIMO) technology utilizes antenna arrays on both sides of the link, with tens to hundreds antenna elements, which allows an increase in antenna gain, spatial diversity and spatial multiplexing [1]-[4]. However, with the presence of these structures, several side effects arise, causing the performance of the communications system to be affected. In this context, it is convenient to

"This work was partially funded by Research Support Foundation of Ceará State (FUNCAP) and the Higher Education Personnel Improvement Coordination (Capes), and was also supported in part by the Wireless Telecommunications Research Group (GTEL), Department of Teleinformatics Engineering, Federal University of Ceará, Brazil.”

F. Estevão S. Pereira is with the Wireless Telecommunications Research Group (GTEL), Departament of Teleinformatics Engineering, Federal University of Ceará, Brazil (e-mail: estevaosimao@gtel.ufc.br).

Josef A. Nossek is with the Department of Teleinformatics Engineering, Federal University of Ceará, Fortaleza, Brazil and Department of Electrical and Computer Engineering, Technical University of Munich, Germany (email: josef.a.nossek@gtel.ufc.br).

F. Rodrigo P. Cavalcanti is with the Wireless Telecommunications Research Group (GTEL), Departament of Teleinformatics Engineering, Federal University of Ceará, Brazil (e-mail: rodrigo@gtel.ufc.br)

Digital Object Identifier: 10.14209/jcis.2020.29 analyze and revisit two important concepts in an antenna array: mutual coupling and impedance matching among antenna elements in the array. When utilizing antenna arrays it is important to model these arrays in a physically consistent way. This is best done by a matrix description of the array multiport, which of course depends on the type of antenna elements, their orientation and spacing [5].

The topic of designing multiports for antenna arrays, which provide decoupled and matched ports for the amplifiers (either high power amplifiers (HPA) for transmission or low noise amplifiers (LNAs) for reception), has been treated in the literature by several authors [6]-[7], [8], [9]-[10], [11]-[12]. Some of them are using lumped elements, some are using strip lines, and some use the so-called parasitic antenna.

This paper proposes and evaluates three strategies of joint Decoupling and Impedance Matching Network (DMN) for an array of dipole antennas. From the multiport communication theory presented in [3], the appropriate equations for the proposed DMN are taken. The main contribution of this paper is to offer three possible DMN implementations and compare their performance.

The first method, called DMN with Lumped Elements (DMN-LE), performs the decoupling and impedance matching steps by a lumped circuit with capacitors and inductors. This method can easily generalized to any number $\mathrm{N}$, but practically limited to reasonably low N. The second method, called DMN with Ring Hybrid (DMN-RH), is modeled with a microstrip line in a ring format. The DMN-RH is limited to two antenna elements. The third method, called Networkless Decoupling and Matching (NDM), brings a concept of decoupling and impedance matching without the presence of a multiport network itself. This enables the design of an antenna array that performs DMN operations in a simplified and compact manner. For a fair comparison of the three approaches we restrict the detailed analyses and simulation to an array of only two antennas elements.

This is justified by the fact that the complexity of all DMN designs is unfortunately growing quadratically with the number of antenna elements $N$ (it grows at least with $N^{2}+N$ as shown in [13]), the implementation of DMNs is limited to a low number of antenna elements, say 2, 3 or at most 4 . Nevertheless these small arrays are still useful for massive MIMO, because they pave the way to implement a massively large array with a reasonable large number of such small subarrays, each of them with very few antennas, say 2 or 3 . In this way,within the subarrays, we use very small spacing between antenna elements and decouple them with realizable DMNs, while the spacing between these subarrays should not 
be that small, such that the residual mutual coupling could be ignored.

A case study will be conducted in order to compare the performance results and obtain a DMN structure capable of providing high efficiency to the antenna arrays and communication systems.

The rest of paper is organized as follows. Section II brings the general definition and concept of DMN. Section III presents the DMN-LE along with an analysis of the system. Section IV presents the DMN-RH, explaining the process of its design. Section V demonstrates the networkless decoupling technique along with the impedance matching step. Section VI shows computer simulation results and discusses them. Finally, Section VII summarizes the findings and gives an outlook to future works.

\section{Decoupling And Matching Network (DMN)}

The DMN is a device capable of performing the decoupling operations between the elements of an antenna array and the impedance matching of the connected amplifier ports. The DMN ports are designed to provide an impedance of $50 \Omega$ on the source side. Fig. 1 shows the representation of the $2 \mathrm{~N}$-Port DMN providing decoupled ports matched to the sources, each source with impedance $\mathrm{R}=50 \Omega$.

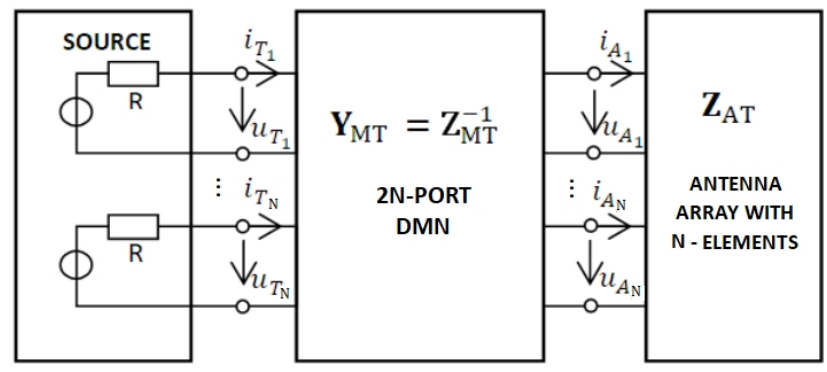

Fig. 1. 2N-Port DMN block representation.

In this representation the source comprised of ports $T_{1}$ to $T_{N}$ represents the insertion of the signal that will be transmitted in each port. On the other side of the DMN, on the ports $A_{1}$ to $A_{N}$, the antenna array with its impedance matrix $\left(\mathbf{Z}_{A T}\right)$ is connected.

Equations (1) and (2) show the definition of the voltage and current vectors at the aforementioned ports:

$$
\begin{gathered}
\boldsymbol{u}_{T}=\left[\begin{array}{c}
u_{T_{1}} \\
\vdots \\
u_{T_{N}}
\end{array}\right], \boldsymbol{i}_{T}=\left[\begin{array}{c}
i_{T_{1}} \\
\vdots \\
i_{T_{N}}
\end{array}\right], \\
\boldsymbol{u}_{A}=\left[\begin{array}{c}
u_{A_{1}} \\
\vdots \\
u_{A_{N}}
\end{array}\right], \boldsymbol{i}_{A}=\left[\begin{array}{c}
i_{A_{1}} \\
\vdots \\
i_{A_{N}}
\end{array}\right] .
\end{gathered}
$$

The purpose of this method is to obtain the matrix $\boldsymbol{Z}_{M T}$ such that the sources achieve power matching and decoupling by the DMN as,

$$
\boldsymbol{u}_{T}=R \cdot \boldsymbol{I} \cdot \boldsymbol{i}_{T},
$$

where $\boldsymbol{I}$ is the identity matrix.

This can be achieved with the following equations:

$$
\begin{gathered}
{\left[\begin{array}{l}
\boldsymbol{u}_{T} \\
\boldsymbol{u}_{A}
\end{array}\right]=\boldsymbol{Z}_{M T}\left[\begin{array}{c}
\boldsymbol{i}_{T} \\
-\boldsymbol{i}_{A}
\end{array}\right],} \\
\boldsymbol{u}_{A}=\boldsymbol{Z}_{A T} \boldsymbol{i}_{A}, \\
\boldsymbol{Z}_{M T}=\left[\begin{array}{cc}
\boldsymbol{0} & \boldsymbol{X}_{1} \\
\boldsymbol{X}_{1} & \boldsymbol{X}_{2}
\end{array}\right]
\end{gathered}
$$

where,

$$
\begin{gathered}
\boldsymbol{X}_{1}=-j \sqrt{R} R e\left\{\boldsymbol{Z}_{A T}\right\}^{\frac{1}{2}} \\
\boldsymbol{X}_{2}=-j \operatorname{Im}\left\{\boldsymbol{Z}_{A T}\right\},
\end{gathered}
$$

from [3] and [14].

The antenna array that will be discussed in this paper is presented in Fig. 2. This array consists of two half-wavelength dipole antennas where the distance between them is given by $\rho$. The feed is also represented in Fig. 2, located at the center between the $\lambda / 4$ segments with its respective voltage $u_{A}$ and current $i_{A}$ for each antenna element.

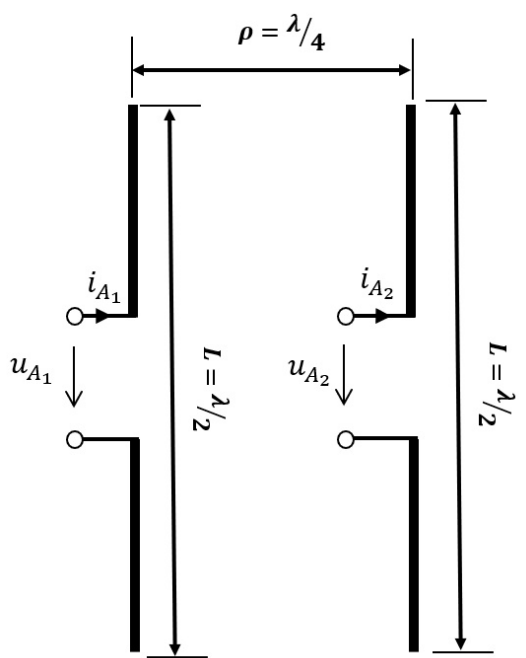

Fig. 2. Dipole Antenna Array with two elements.

\section{DMN WITH LUMPED ELEMENTS (DMN-LE)}

The DMN-LE performs the decoupling between the elements of the antenna array and the impedance matching between the array and the source by means of lumped elements. Lumped elements are widely used in microwave systems to perform impedance transformation [6], [7], [15]-[17].

\section{A. Design of DMN Lumped Elements}

For the detailed design of DMN-LE it is convenient to work with the admittance matrix $\boldsymbol{Y}_{M T}$. Its representation will be referred to as a general 4-port implemented with the reactive elements $Y_{1}$ to $Y_{10}$ according to Fig. 3, when the number of elements of the antenna array is defined as $N=2$. 


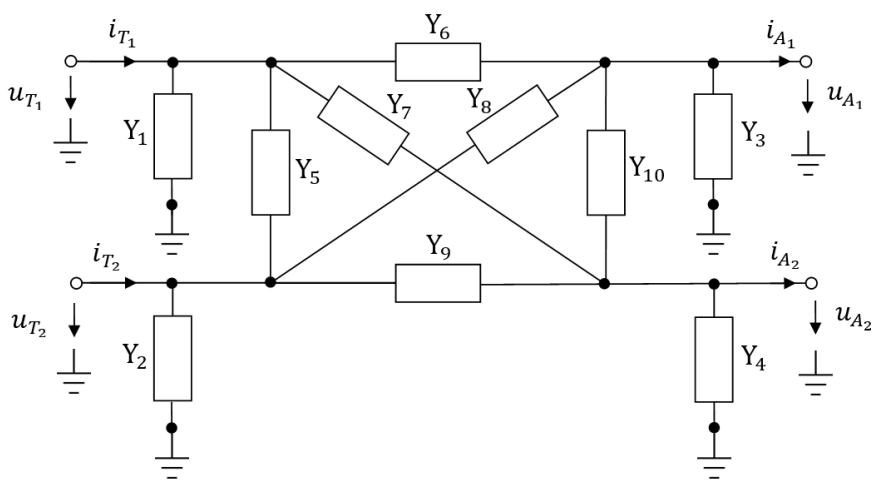

Fig. 3. Circuit diagram for the DMN-LE.

For this diagram, the ports $u_{T_{1}}$ and $u_{T_{2}}$ represent the input ports of the DMN-LE, while $u_{A_{1}}$ and $u_{A_{2}}$ represent the output ports connecting the antenna array. The DMN-LE is designed to operate at a center reference frequency $f_{r}$.

The DMN-LE is initially characterized by $\boldsymbol{Y}_{M T}$ and in order to perform the modeling of each of its elements, it is necessary to know the impedance matrix $\boldsymbol{Z}_{A T}$ of the antenna array. The matrix $\boldsymbol{Y}_{M T}$ is obtained from $\boldsymbol{Z}_{M T}$ by inversion as,

$$
\boldsymbol{Y}_{M T}=\boldsymbol{Z}_{M T}^{-1}=\left[\begin{array}{cc}
\boldsymbol{B}_{1} & \boldsymbol{B}_{2} \\
\boldsymbol{B}_{2} & \boldsymbol{0}
\end{array}\right]
$$

where,

$$
\boldsymbol{B}_{1}=-j \frac{1}{R} \operatorname{Re}\left\{\boldsymbol{Z}_{A T}\right\}^{-\frac{1}{2}} \operatorname{Im}\left\{\boldsymbol{Z}_{A T}\right\} \operatorname{Re}\left\{\boldsymbol{Z}_{A T}\right\}^{-\frac{1}{2}},
$$

and

$$
\boldsymbol{B}_{2}=j \frac{1}{\sqrt{R}} \operatorname{Re}\left\{\boldsymbol{Z}_{A T}\right\}^{-\frac{1}{2}}
$$

The impedance matrix of the array in Fig. 2 has been computed according to [18, Chapter 13] and results in (12).

$$
\boldsymbol{Z}_{A T}=\left[\begin{array}{ll}
73.05+j 42.44 & 40.74-j 28.31 \\
40.74-j 28.31 & 73.05+j 42.44
\end{array}\right] \Omega
$$

at the center frequency $f_{r}=c / \lambda$, where $c$ is the speed of light.

To calculate each element of the DMN-LE presented in Fig. 3, we use the matrix

$$
\boldsymbol{Y}_{M T}=\left[\begin{array}{llll}
y_{11} & y_{12} & y_{13} & y_{14} \\
y_{12} & y_{22} & y_{23} & y_{24} \\
y_{13} & y_{23} & y_{33} & y_{34} \\
y_{14} & y_{24} & y_{34} & y_{44}
\end{array}\right]
$$

where each of its elements is defined as

$$
\begin{array}{r}
Y_{1}=y_{11}+y_{12}+y_{13}+y_{14}, \\
Y_{2}=y_{22}+y_{12}+y_{23}+y_{24}, \\
Y_{3}=y_{33}+y_{13}+y_{23}+y_{34}, \\
Y_{4}=y_{44}+y_{14}+y_{24}+y_{34}, \\
Y_{5}=-y_{12}, \\
Y_{6}=-y_{13}, \\
Y_{7}=-y_{14}, \\
Y_{8}=-y_{23}, \\
Y_{9}=-y_{24}, \\
Y_{10}=-y_{34} .
\end{array}
$$

The conversion of each element of the matrix $\boldsymbol{Y}_{M T}$ into capacitive or inductive elements is performed based on $Y_{i}=j B_{i}$, as shown in (15) and (16). Thus, if $B_{i}>0$,

$$
C_{i}=\frac{B_{i}}{\omega}
$$

and if $B_{i}<0$, we have,

$$
L_{i}=-\frac{1}{B_{i} \omega}
$$

where $\omega=2 \pi f_{r}$.

For the DMN-LE modeling with the addition of losses, the equivalent circuit model employs the concept of Q-factor. In this way, using the Q-factor obtained from the manufacturer of the electronic component, [19] and [20], it is possible to take losses into account.

\section{DMN WITH RING HYBRID (DMN-RH)}

The Ring Hybrid ( $\mathrm{RH}$ ) is a linear microwave device which is generally defined as a 4-port [8]. It is composed of the branch-line coupler design with a $90^{\circ}$ hybrid junction, with spacing of $\lambda / 4$ between ports $T_{1}$ and $A_{2}, T_{1}$ and $A_{1}$, and $A_{1}$ and $T_{2}$. The spacing between ports $T_{2}$ and $A_{2}$ is $3 \lambda / 4$. Such a $\mathrm{RH}$ is readily implemented using microstrip lines as shown in Fig. 4. In relation to obtaining the impedance matching of the ports $T_{1}$ and $T_{2}$, a transmission line segment is added to each of these ports.

In this way, the RH is a useful solution for the DMN allowing the operation of mutual decoupling between the ports and the impedance matching, only with its use.

Fig. 4 shows the standard design of the RH with impedance characteristic $Z_{0}$.

\section{A. Design of the DMN-RH}

The RH modeling is based on formatting the microstrip configuration parameters that will be used. To the design of DMN-RH, it is necessary to define the parameters of the transmission line segments both in the ring itself and at the ports $T_{1}$ and $T_{2}$. Thus, to define the line widths of the ring, the impedance $Z_{0}$, and the port impedances $Z_{1}\left(T_{1}\right)$ and $Z_{2}\left(T_{2}\right)$ are required. The derivation of these parameters is based on the circuit analysis of the RH microstrip according to [8]. For 


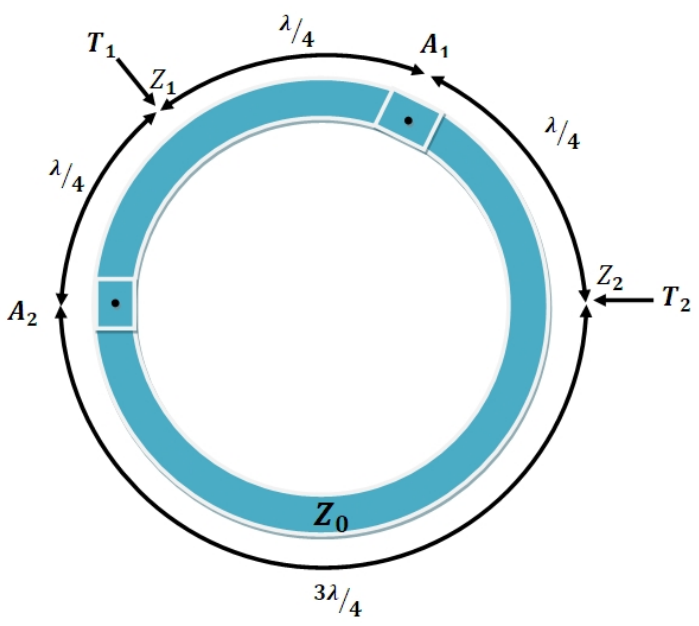

Fig. 4. DMN-RH.

the derivation of parameter $Z_{0}$ (RH characteristic impedance) the equation

$$
Z_{0}=\sqrt[4]{4|a+b||a-b| R^{2}}
$$

is used based on the impedance parameters of the dipole antenna array, the $\boldsymbol{Z}_{A T}$ matrix, defined previously, where $a=Z_{11}=Z_{22}, b=Z_{12}=Z_{21}$.

For the derivation of $Z_{1}$ and $Z_{2}$ of the impedances at $T_{1}$ and $T_{2}$, which still have to be matched to $R$, the following equations are used

$$
\begin{aligned}
& Z_{1}=\frac{Z_{0}^{2} / 2}{a+b}, \\
& Z_{2}=\frac{Z_{0}^{2} / 2}{a-b} .
\end{aligned}
$$

Section VI-C shows how this can be achieved.

\section{Networkless Decoupling ANd Matching (NDM)}

This method aims to achieve the impedance matching and decoupling between the elements of the antenna array without having a multi-port impedance matching/decoupling structure. For this, a new antenna element is inserted equidistantly between the existing elements. Furthermore, 3 linear voltage sources are employed as shown in Fig. 5.

The overall electromagnetic field is a linear superposition of the contributions of all three antenna elements. The excitation voltage of the third antenna element is controlled by the voltages of the other two. This control is designed to let all antenna elements decoupled and matched.

Each port has its voltage phasor given as,

$$
u_{i}=\frac{u_{0, i}}{1+\frac{Z_{i}}{Z_{i}^{*}}}, \quad i=1,2,3 .
$$

The generators must also be designed to have proper output impedance $Z_{i}$.

In summary, the method allows the first two generators to have arbitrary values, while the voltage of the third generator $u_{0,3}$ is controlled by the other two, according to

$$
u_{0,3}=g_{1} u_{0,1}+g_{2} u_{0,2},
$$

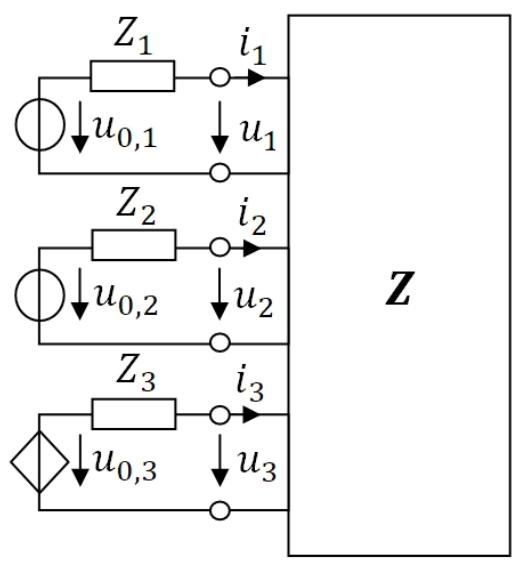

Fig. 5.3 linear generators connected to a linear 3-port.

where $g_{1}$ and $g_{2}$ are properly chosen complex constants.

\section{A. Networkless Matching Design}

For the NDM modeling it is necessary to define the vectors $\boldsymbol{u}=\left[\begin{array}{lll}u_{1} & u_{2} & u_{3}\end{array}\right]^{T}, \boldsymbol{u}_{0}=\left[\begin{array}{lll}u_{01} & u_{02} & u_{03}\end{array}\right]^{T}$ and $\boldsymbol{i}=\left[\begin{array}{lll}i_{1} & i_{2} & i_{3}\end{array}\right]^{T} . \boldsymbol{Z}$ is the impedance matrix of the antenna array consisting of three elements and $\boldsymbol{Z}_{0}$ is the diagonal matrix of the three source impedances, as

$$
\mathbf{Z}=\left[\begin{array}{lll}
a & b & c \\
b & a & c \\
c & c & a
\end{array}\right]
$$

and

$$
\mathbf{Z}_{0}=\left[\begin{array}{ccc}
Z_{1} & 0 & 0 \\
0 & Z_{2} & 0 \\
0 & 0 & Z_{3}
\end{array}\right]
$$

with $a=Z_{11}, b=Z_{12}$ and $c=Z_{13}$.

Requiring power matching as given by (19) and (20) leads to the following derivation [21]:

$$
\left(\mathbf{1}-\mathbf{Z}_{0}\left(\mathbf{Z}_{0}+\mathbf{Z}\right)^{-1}\right)\left[\begin{array}{cc}
1 & 0 \\
0 & 1 \\
g_{1} & g_{2}
\end{array}\right]=\left[\begin{array}{cc}
x_{1} & 0 \\
0 & x_{2} \\
g_{1} x_{3} & g_{2} x_{3}
\end{array}\right]
$$

where,

$$
\begin{aligned}
& x_{1}=\left(1+\exp \left(2 j \arg Z_{1}\right)\right)^{-1}, \\
& x_{2}=\left(1+\exp \left(2 j \arg Z_{2}\right)\right)^{-1}, \\
& x_{3}=\left(1+\exp \left(2 j \arg Z_{3}\right)\right)^{-1} .
\end{aligned}
$$

Thus, given $\boldsymbol{Z}$, we solve the above equation for $\boldsymbol{Z}_{0}, g_{1}$ and $g_{2}$ to have

$$
\begin{gathered}
g_{1}=g_{2}=g=\frac{-c^{2}+\left(a+Z_{3}\right) b}{\left(b-a+Z_{1}\right) c}, \\
Z_{1}=Z_{2}=a^{*}-b^{*}, \\
Z_{3}=a^{*}-\left(\frac{c^{2}}{b}\right)^{*} .
\end{gathered}
$$

Finally we have to transform the impedances $Z_{1}, Z_{2}$ and $Z_{3}$ to the source resistance of $50 \Omega$ by impedance matching twoports (see Fig. 11). 
To verify the performance of the NDM with a computer tool, it is important to make sure that the third port is always driven by the voltage $u_{03}$ as given by [21], because only then all three ports will be power matched to their corresponding sources. This is achieved by inserting Voltage Controlled Voltage Sources (VCVS). The gains $G_{1}$ and $G_{2}$ are determined by the chosen beamforming and $G_{3}$ is generated as a function of $G_{1}$ and $G_{2}$.

This method is somewhat similar to what is called parasitic elements based decoupling [22], in which the auxiliary antenna is short circuited at the feed point and the geometry is optimized to achieve decoupling.

\section{Numerical Results}

Numerical results were obtained to enable a performance comparison between the three concepts in terms of the $S$ parameters, which are scattering parameters. Regarding the metric for the bandwidth, the reference thresholds of $-20 \mathrm{~dB}$ and $-10 \mathrm{~dB}$ will be considered. The following simulation conditions are assumed:

- Two-element antenna array with half-wavelength dipoles $L=\lambda / 2$.

- Distance between main antennas: $d=\lambda / 4$.

- Reference carrier frequency: $f_{r}=3 G H z$.

- Impedance matrix with a classical model computed according to [18, Chapter 13] .

- Frequency range of interest (for measuring bandwidth): $2.9 \mathrm{GHz}-3.1 \mathrm{GHz}$.

The main objective of this analysis is to verify the behavior of the mutual coupling $\left(S_{12}\right.$ and $\left.S_{21}\right)$ and impedance matching $\left(S_{11}\right.$ and $S_{22}$ ) of the proposed DMN approaches over the frequency range of interest. Circuit simulations employ the ANSOFT DESIGNER SV Software. It is important to note that for this analysis the antenna array used has the beamforming in the end-fire direction. Thus, this research analyzes DMN structures directed to the array of antennas with their elements in parallel.

\section{A. Baseline Performance}

The performance of the antenna array without DMN is presented in Fig. 6. It shows that the resonance frequency performance is around $2.7 \mathrm{GHz}$ (i.e., $300 \mathrm{MHz}$ off the reference frequency). Impedance matching $\left(S_{11}\right.$ or $\left.S_{22}\right)$ has value of around $-26 \mathrm{~dB}$, at resonance frequency, while for the reference frequency a value of $-6 \mathrm{~dB}$ is observed. $S_{12}$ and $S_{21}$ assume a value of around $-12 \mathrm{~dB}$ at the reference frequency. A reference level of $-10 \mathrm{~dB}$ is considered for evaluating the system bandwidth from the $S$ parameters.

\section{B. Results for DMN-LE}

The circuit equivalent of the DMN-LE is shown in Fig. 7. The values of the components are described in Table I, in which the Q-factor values are given according to [19] and [20].

The behavior of the DMN-LE versus the baseline is presented in Fig. 8a, for $S_{11}$ and $S_{22}$. Fig. 8b shows the performance for $S_{12}$ and $S_{21}$.

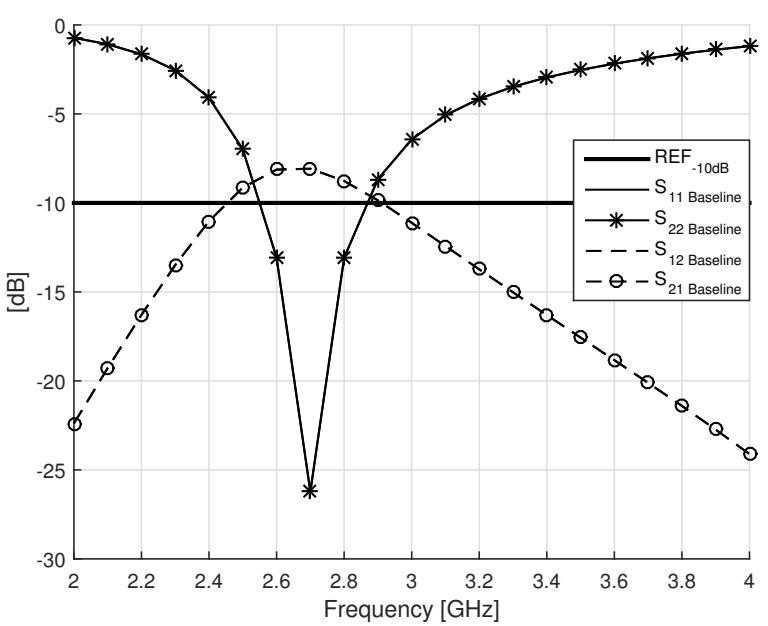

Fig. 6. Baseline array model.

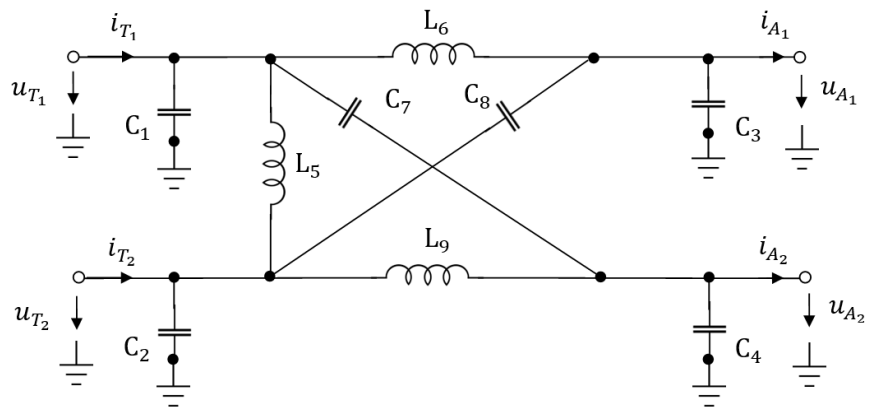

Fig. 7. DMN-LE circuit .

TABLE I

Values of components.

\begin{tabular}{l|c}
\hline \multicolumn{1}{c|}{ Components } & Values Obtained \\
\hline \hline$C_{1}, C_{2}$ & $0.53888 \mathrm{pF}$ \\
& $\mathrm{Q}=137, f_{Q}=2.4 \mathrm{GHz}$ \\
\hline$C_{3}, C_{4}$ & $0.63942 \mathrm{pF}$ \\
& $\mathrm{Q}=131, f_{Q}=2.4 \mathrm{GHz}$ \\
\hline$L_{5}$ & $2.3544 \mathrm{nH}$ \\
& $\mathrm{Q}=64.4, f_{Q}=2.4 \mathrm{GHz}$ \\
\hline$L_{6}, L_{9}$ & $2.7827 \mathrm{nH}$ \\
& $\mathrm{Q}=78.9, f_{Q}=2.4 \mathrm{GHz}$ \\
\hline$C_{7}, C_{8}$ & $0.2975 \mathrm{pF}$ \\
\hline
\end{tabular}

By having both decoupling and impedance matching better than $-20 \mathrm{~dB}$, a bandwidth of $100 \mathrm{MHz}$ is achieved. For a behavior of $-10 \mathrm{~dB}$, a bandwidth of more than $300 \mathrm{MHz}$ is reached.

With these results, subarray modeling is well understood in order to achieve a performance increase of around 500\% compared to the baseline results at the reference frequency for $S_{11}$. For $S_{12}$, there is an increase of $150 \%$ compared to the baseline results at the reference frequency.

Comparing the results obtained in this method with others publications, it is possible to realize that the proposed modeling produces important results, in order to meet the necessary requirements, both in mutual coupling and impedance match- 


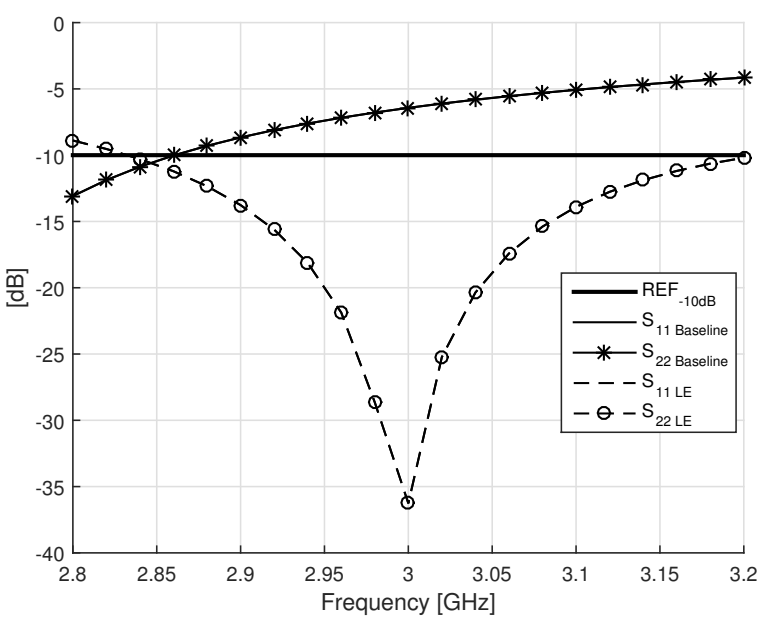

(a)

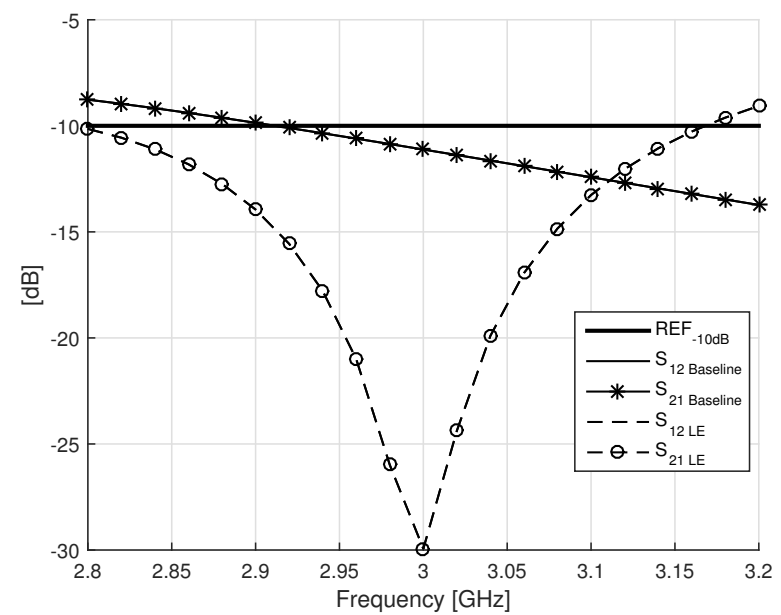

(b)

Fig. 8. DMN-LE versus baseline array.

ing. In [23], for example, a DMN network is presented which shows an increase of $18 \%$ in the return loss bandwidth by $-10 \mathrm{~dB}$. With DMN-LE, a $40 \%$ increase from $-9.8 \mathrm{~dB}$ to $-14 \mathrm{~dB}$ in the main frequency and a greater bandwidth range were observed. In [10], another DMN is shown which presents a 10 element DMN circuit. Therefore, a simplification in the DMN-LE can be observed by reducing the number of elements and especially in this case, a single element is eliminated.

In [13] a general method is outlined to design DMNs for arbitrary number of antenna elements. For a simple design approach the number of necessary circuit elements is given by $2 N^{2}+N$, which can be reduced to $N^{2}+N$ with a more elaborate technique. The design method, which has been used here is somewhat in between with $\frac{3}{2}\left(N^{2}+N\right)$. Anyway, unfortunately the number of needed circuit elements grows quadratically with the number of antenna elements restricting the method to a moderately low number of antenna elements.

\section{Results for DMN-RH}

The values obtained from the equations presented for the DMN-RH are show in Table II. Based on these values, the microstrip parameters are obtained.

To obtain the width and length from the impedance value and the electric length, ANSOFT DESIGN SV's microstrip line calculator is used [24]. The substrate RO3006 material is used [25], which has $\epsilon_{r}=6.15$ and the thickness of the substrate is $1.52 \mathrm{~mm}$. The thickness of the strip line and the ground plane is $35 \mu \mathrm{m}$.

TABLE II

DMN-RH Calculated Impedance Parameters.

\begin{tabular}{l|l}
\hline \multicolumn{1}{c|}{ Parameters } & Values Obtained \\
\hline \hline$Z_{0}$ & $97.1845 \Omega$ \\
\hline$Z_{1}$ & $(40.8666-j 5.0754) \Omega$ \\
\hline$Z_{2}$ & $(25.2097-55.2266) \Omega$ \\
\hline
\end{tabular}

Now the ports $T_{1}$ and $T_{2}$ have to be matched to the source resistance of $50 \Omega$. At port $T_{1}$ a strip line is connected with characteristic impedance

$$
Z_{01}=\sqrt{R \cdot \operatorname{Re}\left\{Z_{1}\right\}-\frac{R \cdot \operatorname{Im}\left\{Z_{1}\right\}^{2}}{R-\operatorname{Re}\left\{Z_{1}\right\}}}
$$

providing the desired transformed port resistance of $50 \Omega$ and electric length $\theta_{1}=\frac{2 \pi}{\lambda} l_{1}$ with

$$
l_{1}=\frac{\lambda}{2 \pi} \cdot \arctan \left(\frac{R \cdot \operatorname{Re}\left\{Z_{1}\right\} \cdot Z_{01}}{R \cdot \operatorname{Im}\left\{Z_{1}\right\}}\right) .
$$

Unfortunately $T_{2}$ could not be matched in the same way with a single strip line. First the reactive part of $Z_{2}$ is eliminated by a strip line with $Z_{21}=50 \Omega$ and appropriate length $l_{21}$. Then a strip line with length $\lambda$ and appropriate characteristic impedance $Z_{22}$ is used leading to design values given in Table III. The layout for this RH with the matching strip lines for port $T_{1}$ and $T_{2}$ is given in Fig. 9.

TABLE III DMN-RH Design Parameters.

\begin{tabular}{l|l}
\hline \multicolumn{1}{c|}{ Parameters } & Values Obtained \\
\hline \hline$\theta_{r}$ & $90^{\circ}$ \\
$l_{r}$ & $12.5859 \mathrm{~mm}$ \\
$w_{r}$ & $0.4356 \mathrm{~mm}$ \\
\hline$Z_{01}$ & $43.6155 \Omega$ \\
$\theta_{1}$ & $122.498^{\circ}$ \\
$l_{1}$ & $15.8662 \mathrm{~mm}$ \\
$w_{1}$ & $2.7999 \mathrm{~mm}$ \\
\hline$Z_{21}$ & $50 \Omega$ \\
$\theta_{21}$ & $51.056^{\circ}$ \\
$l_{21}$ & $6.7001 \mathrm{~mm}$ \\
$w_{21}$ & $2.2016 \mathrm{~mm}$ \\
\hline$Z_{22}$ & $23.3544 \Omega$ \\
$\theta_{22}$ & $90^{\circ}$ \\
$l_{22}$ & $11.0431 \mathrm{~mm}$ \\
$w_{22}$ & $7.0753 \mathrm{~mm}$ \\
\hline
\end{tabular}

The bandwidth for decoupling $\left(S_{21}\right.$ and $\left.S_{12}\right)$ better than $-20 \mathrm{~dB}$ is larger than $400 \mathrm{MHz}$, for matching at port $T_{1}\left(S_{11}\right)$ 


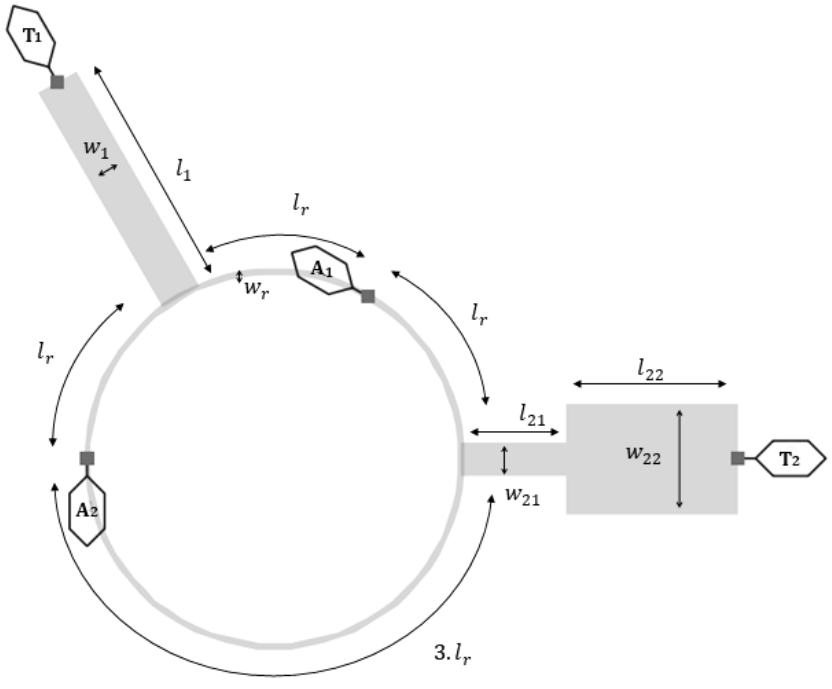

Fig. 9. DMN-RH Design.

better than $-20 \mathrm{~dB}$ is $250 \mathrm{MHz}$, while matching at port $T_{2}$ $\left(S_{22}\right)$ is the bottleneck. Here a bandwidth of only $100 \mathrm{MHz}$ for being better than $-10 \mathrm{~dB}$ and less than $50 \mathrm{MHz}$ for better than $-20 \mathrm{~dB}$ is achieved.

This result shows an increase of around $700 \%$ to $800 \%$ compared to the baseline results for $S_{11}$ and $S_{12}$, respectively, in the reference frequency. Similarly, there is an improvement in bandwidth mainly for the $S_{11}$, with a better result than the defined bandwidth, below $2.9 \mathrm{GHz}$ and above $3.1 \mathrm{GHz}$, considering a threshold $-10 \mathrm{~dB}$. In the same way, $S_{12}$ and $S_{21}$ shows a behavior with an increase of around $550 \%$, at the main frequency. Therefore, according to the observation of these behaviors, it is possible to notice that this structure, despite having a physical limitation on the number of elements, has positive premises for the modeling of antenna subarrays.

In this analysis, when compared to [11] and [26], it is possible to observe that the proposed structure provides better yields, both in the coupling and impedance matching for the used antenna array. In [26], it can be observed that in both behaviors, $S_{11}$ and $S_{22}$ have a limited bandwidth. For the DMN-RH, it is observed a wider bandwidth mainly for $S_{22}$ and $S_{12}$, which demonstrates better behaviors among the modeling.

\section{Results for Networkless Decoupling and Matching}

The results obtained from (19) to (27) for the NDM modeling are presented in the Tables IV and V, as well as can be visualized in Fig. 11, in which the circuit analysis for the $\mathrm{NDM}$ is presented. It is formed by an AC voltage source with a polar voltage with magnitude of $1 V$ and phase of $0^{\circ}$, and connected to three VCVSs each instantiated by the respective values of $\boldsymbol{u}_{0}^{\prime}$.

The VCVSs are connected to LC-twoports, which provide the impedance matching from $50 \Omega$ to the required impedances $Z_{1}, Z_{2}$ and $Z_{3}$ given in Table IV. The conversion from $\boldsymbol{u}_{0}$ to $\boldsymbol{u}_{0}^{\prime}$ compensates for the magnitude and phase changes caused by these matching twoports.

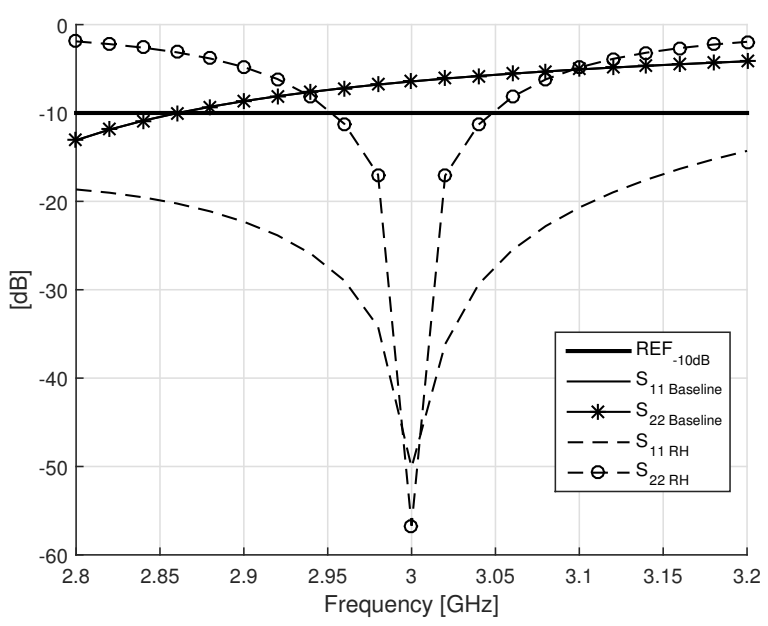

(a)

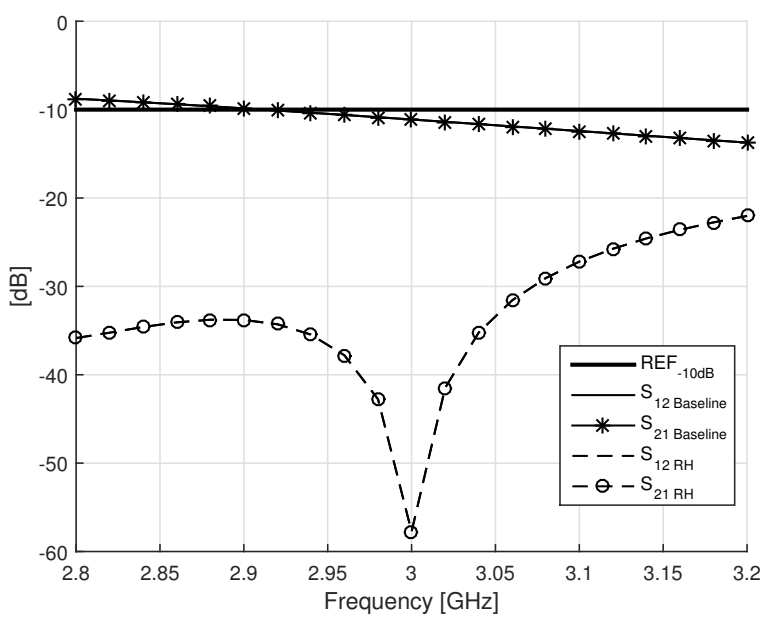

(b)

Fig. 10. DMN-RH versus Baseline Array.

TABLE IV

Values for $\boldsymbol{Z}$ and $\boldsymbol{Z}_{o}$.

\begin{tabular}{l|l}
\hline Parameters & Values Obtained $(\Omega)$ \\
\hline \hline$a$ & $73.05+j 42.44$ \\
$b$ & $40.74-j 28.31$ \\
$c$ & $64.11-j 0.074$ \\
\hline$Z_{1}$ & $32.3-j 70.76$ \\
$Z_{2}$ & $32.3-j 70.76$ \\
$Z_{3}$ & $4.09+j 4.66$ \\
\hline
\end{tabular}

Fig. 12 shows that the bandwidth for decoupling being better than $-20 \mathrm{~dB}$ is quite large (more than $250 \mathrm{MHz}$ ), for matching the achievable bandwidth is less than $100 \mathrm{MHz}$ for better than $-20 \mathrm{~dB}$ and more than $200 \mathrm{MHz}$ for $-10 \mathrm{~dB}$.

The results show an increase of around $433 \%$ and $466 \%$ in $S_{11}$ and $S_{22}$, respectively, at the reference frequency, compared to the baseline results and a better bandwidth mainly in the $S_{22}$, reaching good values above $3.1 \mathrm{GHz}$, at the threshold of $-10 \mathrm{~dB}$. Moreover, it is observed that the third antenna included by the method has decoupling values with the other 


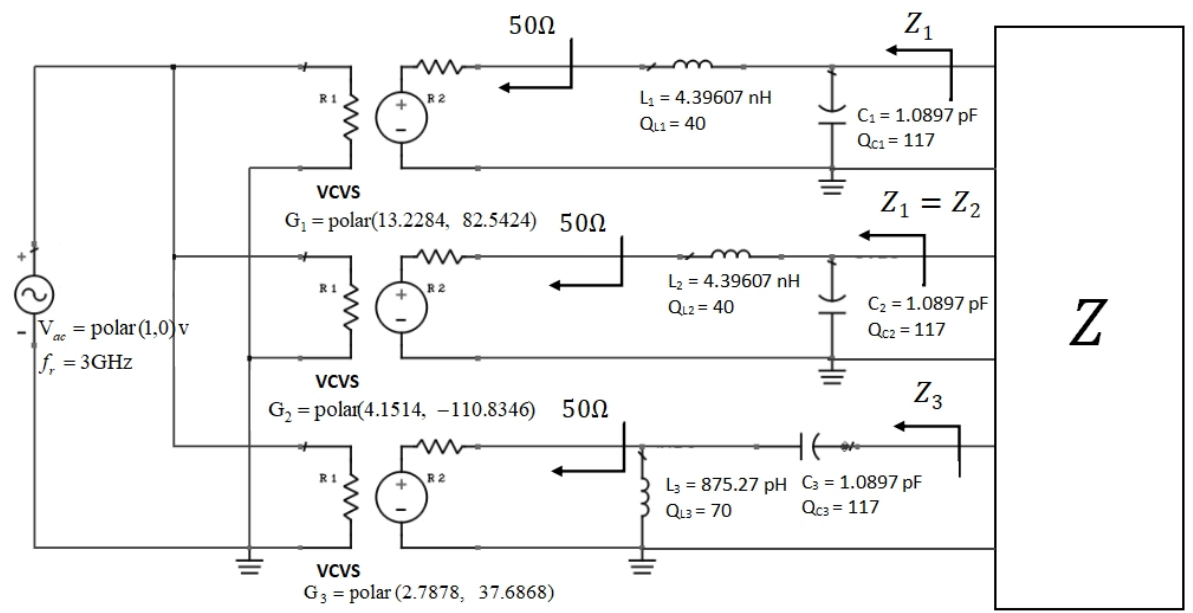

Fig. 11. NDM circuit.

TABLE V

NDM Design Parameters.

\begin{tabular}{l|l}
\hline Parameters & Values Obtained (V) \\
\hline \hline$u_{0,1}$ & $10.6327 \mathrm{e}^{-j 41.8153^{\circ}}$ \\
$u_{0,2}$ & $3.368 \mathrm{e}^{j 124.8037^{\circ}}$ \\
$u_{0,3}$ & $0.8736 \mathrm{e}^{j 109.4254^{\circ}}$ \\
\hline$u_{0,1}^{\prime}$ & $13.2283 \mathrm{e}^{j 82.5424^{\circ}}$ \\
$u_{0,2}^{\prime}$ & $4.1514 \mathrm{e}^{-j 110.8386^{\circ}}$ \\
$u_{0,3}^{\prime}$ & $2.7878 \mathrm{e}^{j 37.6868^{\circ}}$ \\
\hline$g_{1}, g_{2}$ & $0.1176 \mathrm{e}^{j 145.2833^{\circ}}$ \\
\hline
\end{tabular}

antennas around $-40 \mathrm{~dB}$, showing a weak influence among the others, and not degrading the general behavior of the subarray. For $S_{12}$ and $S_{21}$, it is observed an improvement of around $344 \%$ at the main frequency, compared to the baseline results. Therefore, this method shows its ability to perform decouple and impedance matching operations in subarray modeling.

\section{E. Comparison Among DMN approaches}

Fig. 13 shows the graph of comparison among each one of the three proposed DMN methods. In this way, it is possible to perceive the behavior improvement in each method. The NDM method stands out for having the best improvement at the reference frequency for $S_{11}$ and $S_{12}$.

Comparing the results in Fig. 13, it can be seen that, as far as matching $\left(S_{11}, S_{22}\right)$ is concerned, the DMN-LE has the best performance with a $-20 \mathrm{~dB}$ bandwidth of $100 \mathrm{MHz}$, while the DMN-RH is falling short with $\mathrm{a}-20 \mathrm{~dB}$ bandwidth of about $50 \mathrm{MHz}$, and the NDM is in between with about $70 \mathrm{MHz}$ of bandwidth. As far as decoupling $\left(S_{12}, S_{21}\right)$ is concerned, the DMN-RH is the best option with very large $-20 \mathrm{~dB}$ bandwidth of more than $400 \mathrm{MHz}$, followed by the NDM with more than $300 \mathrm{MHz}$ and finally the DMN-LE with around $90 \mathrm{MHz}$ of bandwidth. Summarizing, the NDM seems to present a good compromise.

Once these results are obtained, it can be understood that the modeling of subarrays by each of these methods leads to very reliable performances even considering their limitations regarding the composition of each subarray.

\section{CONCLUSION}

The analysis carried out in this paper described three methods for impedance matching and decoupling for a baseline antenna array in which the scattering parameters $\left(S_{11}, S_{22}\right.$, $S_{12}$ and $S_{21}$ ) were investigated for the $2.9 \mathrm{GHz}$ to $3.1 \mathrm{GHz}$ frequency band (200 MHz bandwidth) around the reference frequency of $3 \mathrm{GHz}$.

With the DMN-LE method, it can be observed that, despite the favorable behavior with respect to the baseline, it demands a complex circuit structure which makes its design difficult for compact applications. On the other hand, the DMNRH method presented a different performance in terms of bandwidth for each of its ports which reduces its overall bandwidth. Therefore, it ends up being discarded in favor of a DMN solution that offers the same behavior in both output ports. Finally the NDM method showed excellent performance, enabling a compact and efficient antenna system for the impedance matching and the minimization of the destructive effects of coupling between the elements of the proposed antenna array.

Therefore, this proposed method has as its main perspective the modeling of multiport array not limited to a 2-element arrays, as in [12]. However when adding more than two antennas the method becomes obviously more complex. With this understanding, modeling and development of subarray structures is the applicable solution which allows the composition of MIMO antenna arrays so that the distance between each subarray is sufficiently capable of guaranteeing the noninteraction between the subarrays, allowing the total composition in a single antenna array.

Therefore, for the overall conclusion, it is possible to notice that from a impedance matching point of view, the DMNLE provides the highest bandwidth, while, from a decoupling point of view, the DMN-RH is the best solution and the NDM is in between the two aspects. Thus, the choice for the best 


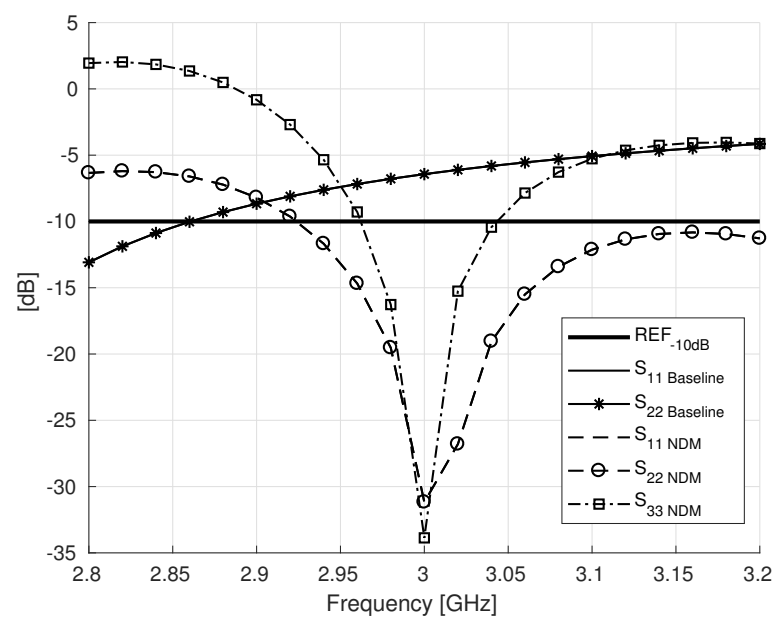

(a)

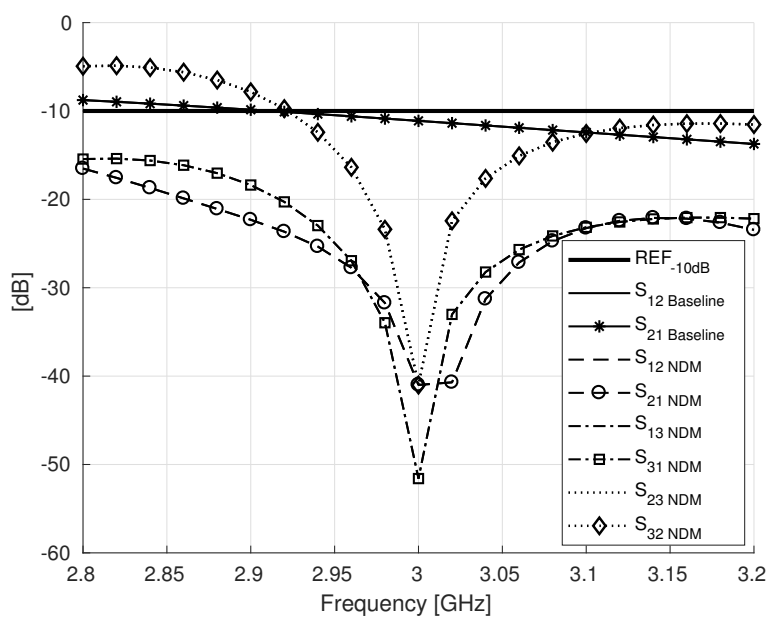

(b)

Fig. 12. NDM versus baseline array.

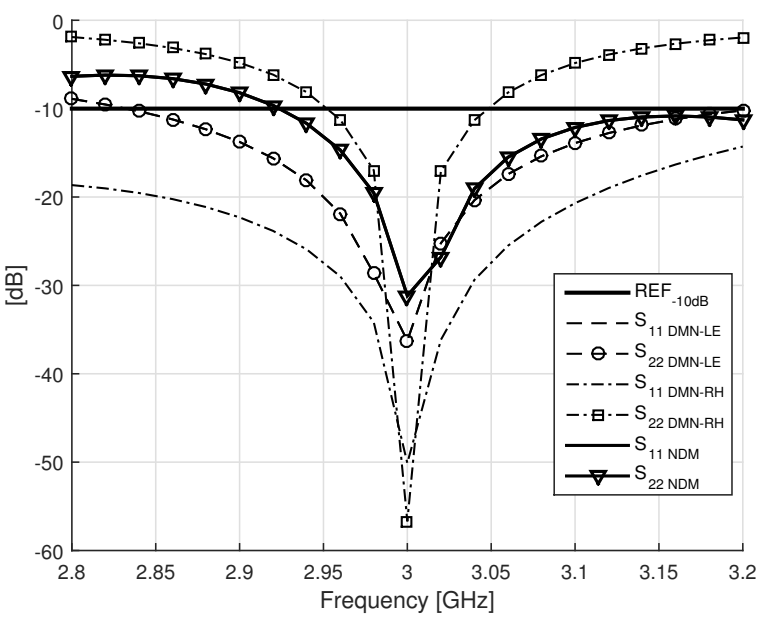

(a)

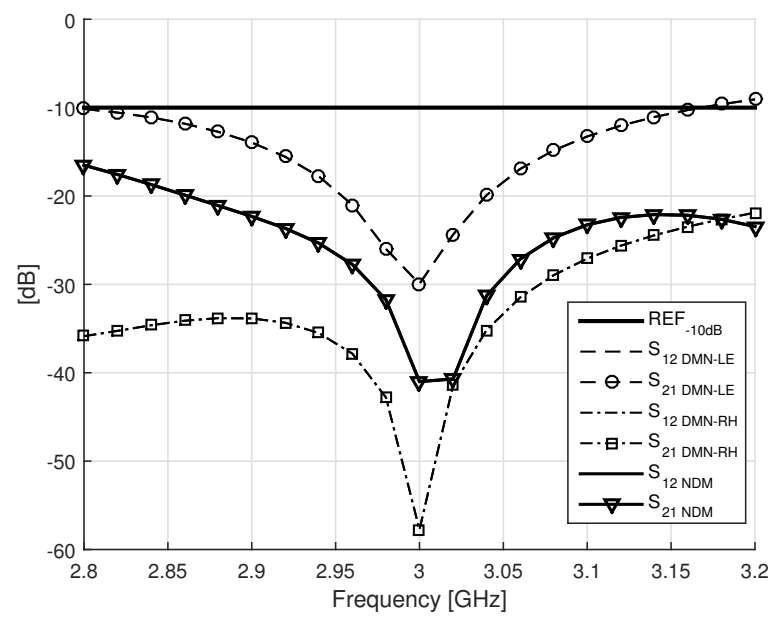

(b)

Fig. 13. Comparison of analysis parameters: $S_{11}, S_{22}, S_{12}$ and $S_{21}$.

method will depend on the specific requirements, in which one of the three best suits.

As future works, the analysis of the NDM regarding the gain and beamforming of the antenna array can be investigated; the analysis of a three element antenna array can be performed; and a generalization of this formulation to more elements of an antenna array can be proposed.

\section{REFERENCES}

[1] B. Yang, Z. Yu, Y. Dong, J. Zhou, and W. Hong, "Compact tapered slot antenna array for $5 \mathrm{~g}$ millimeter-wave massive mimo systems," IEEE Transactions on Antennas and Propagation, vol. 65, no. 12, pp. 67216727, 2017, doi: 10.1109/TAP.2017.2700891.

[2] F. Wang, S. Li, Q. Zhou, and Y.-B. Gong, "Compact wideband quadelement mimo antenna with reversed s-shaped walls," Progress In Electromagnetics Research M, vol. 78, pp. 193-201, 01 2019, doi: 10.2528/PIERM19010201.

[3] M. T. Ivrlač and J. A. Nossek, "The multiport communication theory," IEEE Circuits and Systems Magazine, vol. 14, no. 3, pp. 27-44, 2014, doi: 10.1109/MCAS.2014.2333618.
[4] L. Lu, G. Y. Li, A. L. Swindlehurst, A. Ashikhmin, and R. Zhang, "An overview of massive mimo: Benefits and challenges," IEEE Journal of Selected Topics in Signal Processing, vol. 8, no. 5, pp. 742-758, 2014, doi: 10.1109/JSTSP.2014.2317671.

[5] C. Balanis, Antenna theory: analysis and design. Wiley-Interscience, 2005.

[6] B. S. Yarman, M. Sengul, and A. Kilinc, "Design of practical matching networks with lumped elements via modeling," IEEE Transactions on Circuits and Systems I: Regular Papers, vol. 54, no. 8, pp. 1829-1837, Aug 2007, doi: 10.1109/TCSI.2007.902411.

[7] M. Sengül, "Broadband single matching with lumped elements," in 2015 9th International Conference on Electrical and Electronics Engineering (ELECO), Nov 2015, pp. 115-118, doi: 10.1109/ELECO.2015.7394554.

[8] R. E. Collin, "Foundation of microwave engineering," IEEE Press Series Electromagn Wave Theory, 01 1966, doi:10.1109/9780470544662.

[9] S. Salama and A. Abuelhaija, "Parasitic element based decoupling network for a two-element mri phased array," 04 2019, pp. 790-793, doi:10.1109/JEEIT.2019.8717525.

[10] L. Li, S. Venkatasubramanian, A. Lehtovuori, C. Icheln, M. Heino, and K. Haneda, "T-shaped decoupling network for wideband isolation improvement between two strongly coupled antennas," in 2015 Loughborough Antennas Propagation Conference (LAPC), 2015, pp. 1-4, doi: 10.1109/LAPC.2015.7366140. 
[11] Q. Gong, Y.-C. Jiao, and S.-X. Gong, "Compact mimo antennas using a ring hybrid for wlan applications," Journal of Electromagnetic Waves and Applications, vol. 25, pp. 431-441, 04 2012, doi: $10.1163 / 156939311794362939$.

[12] X. Zou, G. Wang, Y. Wang, and H. Li, "An efficient decoupling network between feeding points for multielement linear arrays," IEEE Transactions on Antennas and Propagation, vol. 67, no. 5, pp. 31013108, 2019, doi: 10.1109/TAP.2019.2899039.

[13] D. Nie, B. Hochwald, and E. Stauffer, "Electromagnetic decoupling and complexity," in 2014 Information Theory and Applications Workshop (ITA), 2014, pp. 1-6, doi: 10.1109/ITA.2014.6804229.

[14] M. T. Ivrlač and J. A. Nossek, "Toward a circuit theory of communication," IEEE Transactions on Circuits and Systems I: Regular Papers, vol. 57, no. 7, pp. 1663-1683, 2010, doi: 10.1109/ICEAA.2009.5297300.

[15] G. P. Riblet, "A matched lumped element five-port which is based on the comparator circuit," IEEE Transactions on Circuits and Systems, vol. 35, no. 10, pp. 1307-1311, Oct 1988, doi: 10.1109/31.7604.

[16] J. N. H. Wong and C. S. Aitchison, "The bandwidth benefits of close-to-chip lumped element matching of microwave high power amplifiers," in 6th IEEE High Frequency Postgraduate Colloquium (Cat. No.01TH8574), Sep. 2001, pp. 147-151, doi: 10.1109/HFPSC.2001.962186.

[17] W. Wu and B. Guan, "A low-profile broadband uhf base station antenna based on lumped-element matching network," in 2016 11th International Symposium on Antennas, Propagation and EM Theory (ISAPE), Oct 2016, pp. 9-12, doi: 10.1109/ISAPE.2016.7833886.

[18] S. A. Schelkunoff and H. T. Friis., Antennas: Theory and Practice. New York, Wiley-Interscience, 1952, vol. 116, doi: 10.1126/science.116.3010.263-a.

[19] (2019) Avx corporation: Avx rf thin-film rf/microwave capacitor technology - accu-p. [Online]. Available: http://datasheets.avx.com/Accu-P.pdf

[20] (2019) Avx corporation: Avx chip inductors. [Online]. Available: http://catalogs.avx.com/RF-Microwave.pdf.

[21] M. T. Ivrlač, Bernhard Lehmeyer, and J. A. Nossek, "Networkless active power-matching," Internal Report - Technische Universität Mnchen, Tech. Rep., 2014.

[22] S. Salama and K. Solbach, "Parasitic elements based decoupling technique for monopole four square array antenna," in 2014 44th European Microwave Conference. IEEE, 2014, pp. 1504-1507, doi: 10.1109/EuMIC.2014.6997918.

[23] X. Tang, K. Mouthaan, and J. C. Coetzee, "Tunable decoupling and matching network for diversity enhancement of closely spaced antennas," IEEE Antennas and Wireless Propagation Letters, vol. 11, pp. 268-271, 2012, doi: 10.1109/LAWP.2012.2188773.

[24] Ansoft-Designer. Microstrip transmission line. [Online]. Available: http://www.mweda.com/designer/ansoftdesigner/trl/MicrostripTransmissionLine.htm

[25] R. Corporation. (2018) Ro3000 series circuit materials. [Online]. Available: https://www.rogerscorp.com/documents/722/acs/RO3000Laminate-Data-Sheet-RO3003-RO3006-RO3010-RO3035.pdf

[26] Y. Cai and Y. J. Guo, "A frequency-agile compact array with a reconfigurable decoupling and matching network," IEEE Antennas and Wireless Propagation Letters, vol. 10, pp. 1031-1034, 2011, doi: 10.1109/LAWP.2011.2168937.

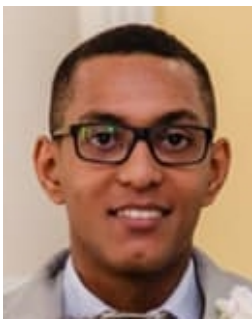

F. Estevão S. Pereira received his B.S. degree in Telecomunication Engineering from Federal Institute of Ceará (IFCE), Brazil, in 2015 and the M.S. degree in eletrical engineering from Military Engineering Institute (IME), Brazil, in 2017. He is currently pursuing the Ph.D. degree in Teleinformatic Engineering at University of Ceará (UFC), Brazil. During his studies, he was supported by the Brazilian agency FUNCAP. He is interested in applied electromagnetism, propagation and antennas, as well as in the development of telecommunications systems. He is a member of the Wireless Telecommunications Research Group (GTEL) working on massive MIMO and MIMO antennas for 5G system.

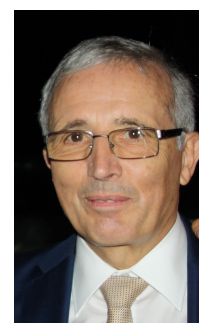

Josef A. Nossek (S'72-M'74-SM'81-F'93-LF'13) received the Dipl.-Ing. and Dr. Techn. degrees in electrical engineering from the University of Technology, Vienna, Austria, in 1974 and 1980, respectively, and the Honorary Doctorate degree in 2013. In 1974, he joined Siemens AG, Munich, Germany, as a Member of the Technical Staff, where he became a Supervisor in 1978 and has also been the Head of Department since 1980. In 1987, he was promoted to be the Head of all radio systems design. From 1989 to 2016, he was a Full Professor for circuit theory and signal processing with the Munich University of Technology (TUM). He was the President Elect and the President of the IEEE Circuits and Systems Society in 2001, 2002, and 2003, respectively. He was a Vice President of Verband der Elektrotechnik, Elektronik und Informationstechnik e.V. (VDE) in 2005 and 2006, the President of VDE in 2007 and 2008 and again the Vice President in 2009 and 2010. Since 2016, he has been an Emeritus of Excellence of TUM and a Full Professor with the Federal University of Ceará, Brazil. He was a recipient of the ITG Best Paper Award in 1988, the Mannesmann Mobilfunk (now Vodafone) Innovation Award in 1998, and the Order of Merit of the Federal Republic of Germany in 2008. He received the Award for Excellence in Teaching from the Bavarian Ministry for Science, Research and Art in 1998, the Golden Jubilee Medal for Outstanding Contributions to the Society from the IEEE Circuits and Systems Society in 1999, the Education Award from the IEEE Circuits and Systems Society in 2008, and the Ring of Honor from VDE in 2014. He was an elected member of the German National Academy of Engineering Sciences (acatech) in 2009.

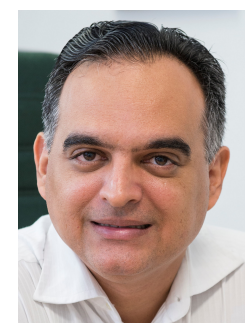

F. Rodrigo P. Cavalcanti received the D.Sc. degree in Electrical Engineering from the State University of Campinas, São Paulo, Brazil, in 1999. Upon graduation, he joined the Federal University of Ceará (UFC), where he is currently an Full Professor and holds the Wireless Communications Chair at the Department of Teleinformatics Engineering. In 2000, he founded, and since then has directed, the Wireless Telecom Research Group (GTEL), which is a research laboratory based on Fortaleza, Brazil, which focuses on the advancement of wireless telecommunications technologies. At GTEL, he manages a 20 -year long program of research projects in wireless communications sponsored by Ericsson Research. In 2017 he was a visiting researcher to Ericsson at main site at Stockholm, Sweden. Prof. Cavalcanti has produced a varied body of work including books, papers, patents and software dealing with wireless access networks and technologies. Prof. Cavalcanti is a distinguished researcher of the Brazilian Scientific and Technological Development Council for his technology development and innovation record. He also holds a Leadership and Management professional certificate from the Massachusetts Institute of Technology, Cambridge, USA 